Review Article

\title{
Chronic Widespread Pain and Fibromyalgia Syndrome: Life-Course Risk Markers in Young People
}

\author{
Aidan C. Tan $\mathbb{D}^{1},{ }^{1}$ Tiina Jaaniste, ${ }^{2,3}$ and David Champion $\mathbb{D}^{2,3}$ \\ ${ }^{1}$ Faculty of Medicine, University of New South Wales, Sydney, New South Wales, Australia \\ ${ }^{2}$ Department of Pain and Palliative Care, Sydney Children's Hospital, Sydney, New South Wales, Australia \\ ${ }^{3}$ School of Women's and Children's Health, University of New South Wales, Sydney, New South Wales, Australia
}

Correspondence should be addressed to David Champion; dchamp@bigpond.net.au

Received 17 February 2019; Accepted 12 March 2019; Published 5 May 2019

Guest Editor: Emilia Matera

Copyright ( 92019 Aidan C. Tan et al. This is an open access article distributed under the Creative Commons Attribution License, which permits unrestricted use, distribution, and reproduction in any medium, provided the original work is properly cited.

\begin{abstract}
Although the life-course concept of risk markers as potential etiological influences is well established in epidemiology, it has not featured in academic publications or clinical practice in the context of chronic widespread pain (CWP) and fibromyalgia syndrome (FMS). Studies of risk markers are required considerations for evaluation of patients and for research because there is no single cause, pathological feature, laboratory finding, or biomarker for CWP or FMS. The early-life risk markers identified by extensive literature review with best evidence for potential causal influence on the development and progression of CWP and FMS include genetic factors, premature birth, female sex, early childhood adversity, cognitive and psychosocial influences, impaired sleep, primary pain disorders, multiregional pain, physical trauma, infectious illness, obesity and inactivity, hypermobility of joints, iron deficiency, and small-fiber polyneuropathy. The case history illustrates the potential etiological influence of multiple risk markers offset by personal resilience.
\end{abstract}

\section{Introduction}

The causal influences on persistent multisite, multiregional, or widespread pain disorders in a young person are often perplexing to physicians and patients. Despite evidence of abnormal nervous system findings, neurophysiological signatures, low-grade systemic inflammation and neuroinflammation, and biochemical alterations in plasma and muscle proteins [1-3], there are no sensitive and specific laboratory findings, biomarkers, or pathological features for chronic widespread pain (CWP) and fibromyalgia syndrome (FMS). Thus, in the absence of a single cause, the etiological focus shifts towards risk markers, defined by Burt [4], as attributes or exposures that are associated with an increased probability of disease, but do not necessarily constitute a causal factor. The conservative term risk marker is preferred to risk factor, association, or comorbidity in this context. Few risk markers for CWP and FMS have been empirically demonstrated to be causal, and there is uncertainty in the literature about whether a risk factor should be a causal link in the etiological chain or may be peripherally associated with an outcome. There is also uncertainty in the literature about what strength of association is needed for an attribute or exposure to be a risk factor for an outcome and how directly it needs to be associated with the outcome.

In this review, the risk markers of CWP and FMS in a young person were considered in a clinical vignette and from a life-course perspective. The life-course approach has demonstrated the influence of early-life events across various health outcomes, including pain $[5,6]$. Identifying risk markers enables a shared understanding of the biopsychosocial antecedents of CWP and FMS and guides early identification, risk prediction, and targeted prevention. Ultimately, the hope is that a life trajectory towards chronic pain can be prevented or interrupted.

In young children, the most common pain disorders without overt disease or trauma-termed idiopathic, nonspecific, functional, or primary-are growing pains, recurrent abdominal pain, and headaches [7]. By age 14, the 
most common pain disorders are headache, abdominal pain, and back pain [8], and children with one pain disorder not infrequently experience multiple other pain disorders [915]. These childhood pain disorders are antecedents of chronic pain in adolescence and adulthood, with long-term persistence, particularly of recurrent abdominal pain and low back pain [16]. Predictors of the transition from acute to persistent musculoskeletal pain in children and adolescents in a prospective study are female sex and poorer conditioned pain modulation, while higher depressive symptoms are associated with higher pain-related disability and poorer quality of life [17]. These features, especially collectively, are likely antecedents to CWP.

CWP is defined as pain in upper and lower and right and left quadrants, for more than three months duration [18]. The prevalence of widespread pain in school-aged children is $7-15 \%[19,20]$. The prevalence of chronic multisite pain in female and male adolescents aged $13-19$ is $11.4 \%$ and $3.8 \%$, respectively [21], and in young adults aged 22 is $10.9 \%$ [22]. A twin study by Kato et al. found the overall prevalence of CWP to be $4.1 \%$.

In CWP meeting criteria for FMS, cognitive, affective, and sensory features emerge, with evidence of abnormal brain networks, altered biology of pain processing, and disturbance of neurobiological function. FMS is defined, especially for epidemiological studies, by the presence of CWP accompanied by chronic fatigue, disturbed sleep, and a spectrum of symptoms associated with nociceptive amplification of neural signaling within the central nervous system [14, 23-27]. There are no validated criteria or evidence-based consensus guidelines for the diagnosis of juvenile FMS [28-30]. Ting et al. [31] concluded that the 2010/2011 American College of Rheumatology fibromyalgia criteria [25] had diagnostic applicability in adolescent females; however, there are no published studies of juvenile FMS which have applied the 2016 revisions to the 2010/2011 fibromyalgia criteria [29].

The onset of juvenile FMS is typically in late childhood and early adolescence. The prevalence of juvenile FMS in children and adolescents is estimated at $1.3 \%$, but up to $3.5 \%$ to $6.2 \%$ for those aged between 15 and 19 , and is substantially higher in females than males [32]. Of adolescents with juvenile-onset FMS, more than $80 \%$ demonstrate persistence of pain, physical impairment, emotional impairment, and other symptoms into early adulthood, and half later meet the full criteria for adult FMS [33].

CWP and FMS cases, as defined, lie on a spectrum from primary pain disorders [34] where the recently introduced term nociplastic [35] may be applied, to secondary disorders where a substantial causal risk factor or causal influence is evident, such as injuries in a motor vehicle accident. In some cases, termed primary FMS, there are no obvious peripheral nociceptive generators or life-course risk markers. In such cases, genetic factors and impaired endogenous pain modulation might apply, and primary pain disorders may precede or accompany the FMS. In other cases, risk markers are prominent, as in the clinical vignette.
1.1. Clinical Vignette. At the time of presentation, the patient, a 25-year-old woman employed in a clerical position, reported a history of persistent pains in wide distribution including hands, feet, ankles, knees, hip regions, back, and neck, as well as headaches. She could be "sore" all day, though was typically better in the morning and worse in the evening. The associated symptoms included her feet "going to sleep," coldness and bluish-white discoloration of fingers and toes, fine tremor of hands, minor subjective swelling of hands, minor sleep impairment, waking unrefreshed, self-reported mild cognitive difficulties, and low energy. Her self-assessed mood was "okay," but she acknowledged "a lot of anxiety" with a tendency to panic reactions. The pain had gradually evolved from childhood and had intensified during adolescence. No obvious disease had been identified, and extensive medical consultations and investigations for inflammatory rheumatic disorders had been negative or inconclusive.

The principal findings on examination were mild obesity, fine hand tremor, moderate hypermobility of joints (meeting Beighton score and arthralgia criteria), and widespread deep tenderness (low-pressure pain threshold). On cutaneous somatosensory testing at a time of relative quiescence of symptoms, responses to cutaneous stimuli, including static and repetitive punctate pressure, were normal. Responses to repetitive deep pressure stimuli at the pain threshold, in wide distribution, demonstrated facilitated temporal summation of pain intensity, minor persistence of after-sensations, but no radiation, interpreted as inferring mild generalized central sensitization. The 2016 revised diagnostic criteria for FMS [25-27] were met.

Past medical and family history revealed a remarkable life-course of potential vulnerability factors for chronic pain disorders:

(i) Family history of depression in both parents, significant anxiety in her father, and migraine in her maternal grandfather.

(ii) Born six weeks premature.

(iii) Childhood adversity through parental disharmony and subsequent divorce at 8 years of age.

(iv) Anxiety and depression from childhood. At the time of presentation, anxiety was more prominent than depression, with occasional panic episodes.

(v) Primary (functional) childhood pain disorders, including growing pains and headaches in early childhood, and a diagnosis of common migraine several years later.

(vi) Iron deficiency, though it was unknown whether this dated from infancy.

(vii) Trauma resulting in fracture of radius and ulna at 8 years of age and fracture of an ankle a year or two later without major sequelae.

(viii) Motor vehicle accident with cervical whiplash injury at 9 years of age.

(ix) Hypermobility of joints. 
(x) Severe Streptococcal throat infection at 16 years of age, followed by chronic fatigue from which she had not recovered. This coincided with the exacerbation and chronicity of her pain.

In view of the array of risk markers combining to create a high-risk phenotype for chronic pain disorders, consideration was given as to why the expression of this young woman's chronic pain disorder was relatively mild. It was apparent that she possessed protective factors including emotional intelligence, social support, good employment, and regular exercise.

\section{Methods}

A literature review was performed by searching the electronic bibliographic databases MEDLINE (via Ovid) and EMBASE (via Ovid), using strategies widely used in systematic reviews, from their inception until September 2017. Search terminology was identified through the controlled vocabulary thesauruses Medical Subject Headings (MeSH) and Emtree. To identify the most appropriate $\mathrm{MeSH}$, the $\mathrm{MeSH}$ tree structure was used, and the subject headings "chronic pain," "fibromyalgia," "risk factors," "child," "adolescent," and "young adult" were selected. This method was replicated for Emtree, and identical subject headings were selected. The search was limited to English language publications, but no other filters were applied. Subject headings were combined with the Boolean operators "OR" and "AND" to find their intersection (Table 1).

Citations were screened by title and abstract for relevance to risk markers for CWP or FMS in children, adolescents, or young adults. The full-text publications of relevant abstracts were attained, and the reference lists of relevant studies were manually reviewed. Our primary focus was family, genetic, and pediatric association studies. Studies of risk markers for CWP or FMS in adults were added when it was apparent that many risk markers provide inferences and hypotheses applicable to young adults and adolescents. Studies of associations and comorbidities likely to be secondary to CWP or FMS were excluded, including restless legs syndrome, low bone mineral density, and cardiovascular disease aggravation. Trauma and infection were included because, like most risk markers, they typically have only contributory causal influences.

The literature search identified 475 articles. After excluding 93 duplicates, citation screening of the remaining 382 articles excluded 276 articles based on irrelevance. The full-text publications of the remaining 106 articles were obtained, reviewed, and qualitatively synthesized. To grade the strength of the research evidence, we adapted the modified grading of recommendations assessment, development, and evaluation (GRADE) framework for a narrative synthesis by Huguet et al. [36].

\section{Results}

Table 2 presents the studies of familial and genetic influences on CWP and FMS. Until recently, FMS diagnosis required physical examination, and thus, large population studies
TABLE 1: Search strategy.

\begin{tabular}{|c|c|c|}
\hline$\#$ & Searches & Results \\
\hline \multicolumn{3}{|l|}{ MEDLINE } \\
\hline 1 & Exp chronic pain/ & 9400 \\
\hline 2 & Exp fibromyalgia/ & 7898 \\
\hline 3 & 1 or 2 & 16903 \\
\hline 4 & Exp risk factors/ & 730540 \\
\hline 5 & Exp child/ & 1810174 \\
\hline 6 & Exp adolescent/ & 1905521 \\
\hline 7 & Exp young adult/ & 646590 \\
\hline 8 & 5 or 6 or 7 & 3134864 \\
\hline 9 & 3 and 4 and 8 & 240 \\
\hline 10 & Limit 9 to English language & 230 \\
\hline \multicolumn{3}{|l|}{ EMBASE } \\
\hline 1 & Exp chronic pain/ & 48537 \\
\hline 2 & Exp fibromyalgia/ & 17126 \\
\hline 3 & 1 or 2 & 62529 \\
\hline 4 & Exp risk factor/ & 837344 \\
\hline 5 & Child/ & 1681331 \\
\hline 6 & Adolescent/ & 1453055 \\
\hline 7 & Exp young adult/ & 200970 \\
\hline 8 & 5 or 6 or 7 & 2611330 \\
\hline 9 & 3 and 4 and 8 & 255 \\
\hline 10 & Limit 9 to English language & 245 \\
\hline Initial number of articles & 475 & \\
\hline $\begin{array}{l}\text { After excluding duplicate } \\
\text { articles }\end{array}$ & 382 & \\
\hline $\begin{array}{l}\text { After excluding irrelevant } \\
\text { articles }\end{array}$ & 106 & \\
\hline Final number of articles & 106 & \\
\hline
\end{tabular}

were unavailable. Stronger evidence for familial and genetic risk markers for CWP has come from population-based twin studies.

Table 3 lists the published risk markers for CWP and FMS determined in pediatric studies. These risk markers reflect underlying neurobiological processes which are incompletely understood. Overall, the graded strength of research evidence, determined using the four quality categories defined by the original GRADE framework [65], high, moderate, low, and very low quality, was greater for risk markers for CWP than FMS because of the earlier availability of questionnaire surveys.

Table 4 displays the modifiable and nonmodifiable risk markers for CWP and FMS from adult studies. Generally, the data were applicable to young adults and adolescents. Again, stronger evidence for risk markers applied to CWP.

3.1. Familial and Genetic Vulnerability. In a populationbased twin study [39], Vehof et al. demonstrated that chronic pain syndromes, including CWP, irritable bowel syndrome, chronic pelvic pain, and spinal pain, [40] shared an underlying genetic factor with an estimated heritability of $66 \%$. Vehof and Williams [93] suggested that the once evolutionary advantage of high pain sensitivity may have turned into a genetic predisposition to the neurobiological underpinnings of chronic pain. Parental CWP was shown to increase the risk of CWP in adult offspring, particularly if both parents had CWP, and offspring were obese [41]. 
TABLe 2: Familial and genetic influences for chronic widespread pain and fibromyalgia.

\begin{tabular}{|c|c|c|c|c|c|c|}
\hline Pain context & Type of study & $\begin{array}{l}\text { Population or } \\
\text { clinic }\end{array}$ & $\begin{array}{l}\text { Number of } \\
\text { participants }\end{array}$ & Multivariate analysis & Reference & Overall quality* \\
\hline CWP & Twin study & Population & 44,897 & No & Kato et al. [37] & +++ \\
\hline CWP & Twin study & Population & 926 & Yes & Burri et al. [38] & ++++ \\
\hline CWP & Twin study & Population & 8564 & Yes & Vehof et al. [39] & +++ \\
\hline CWP & Twin study & Population & 2256 & Yes & Malkin et al. [40] & +++ \\
\hline CWP & Prospective population cohort & Population & 6589 & Yes & Zadro et al. [41] & ++++ \\
\hline CWP & Review & N/A & N/A & N/A & Kerr and Burri [42] & N/A \\
\hline FMS & Family study & Clinic & 805 & Yes & Arnold et al. [43] & ++ \\
\hline FMS & Review & N/A & N/A & N/A & Park et al. [44] & N/A \\
\hline
\end{tabular}

*Overall quality: +, very low; ++, low; +++, moderate; ++++, high.

TABLE 3: Risk markers for chronic widespread pain and fibromyalgia in pediatric samples.

\begin{tabular}{|c|c|c|c|c|c|c|c|c|}
\hline Risk marker & Modifiable & $\begin{array}{c}\text { Pain } \\
\text { context }\end{array}$ & Type of study & $\begin{array}{c}\text { Population } \\
\text { or clinic } \\
\end{array}$ & $\begin{array}{l}\text { Number of } \\
\text { participants }\end{array}$ & $\begin{array}{c}\text { Multivariate } \\
\text { analysis }\end{array}$ & Reference & $\begin{array}{l}\text { Overall } \\
\text { quality }^{*}\end{array}$ \\
\hline Female sex & No & CWP & $\begin{array}{c}\text { Prospective } \\
\text { population cohort }\end{array}$ & Population & 1282 & Yes & $\begin{array}{c}\text { Mikkelsson et al. } \\
{[20]}\end{array}$ & +++ \\
\hline \multirow{7}{*}{ Early-life adversity } & \multirow{7}{*}{ Possibly } & CWP & $\begin{array}{c}\text { Prospective } \\
\text { population cohort }\end{array}$ & Population & 7,571 & Yes & $\begin{array}{l}\text { Jones et al. } \\
{[19,45,46]}\end{array}$ & +++ \\
\hline & & CWP & $\begin{array}{l}\text { Prospective } \\
\text { population cohort }\end{array}$ & Population & 7,470 & No & $\begin{array}{l}\text { Jones et al. } \\
{[19,45,46]}\end{array}$ & +++ \\
\hline & & CWP & $\begin{array}{l}\text { Prospective } \\
\text { population cohort }\end{array}$ & Population & 1,440 & Yes & $\begin{array}{l}\text { Jones et al. } \\
{[19,45,46]}\end{array}$ & +++ \\
\hline & & FMS & Case-control & Clinic & 36 & No & $\begin{array}{c}\text { Olivieri et al. } \\
\text { [47] }\end{array}$ & + \\
\hline & & FMS & Case-control & Clinic & 117 & No & $\begin{array}{c}\text { Bohn et al. [48] } \\
\text { Low and }\end{array}$ & + \\
\hline & & FMS & Review & N/A & N/A & N/A & $\begin{array}{c}\text { Schweinhardt } \\
\text { [49] }\end{array}$ & N/A \\
\hline & & FMS & $\begin{array}{l}\text { Case-control } \\
\text { retrospective }\end{array}$ & Clinic & 75 & Yes & Hellou et al. [50] & ++ \\
\hline \multirow{6}{*}{$\begin{array}{l}\text { Psychosocial factors } \\
\text { (i) Intelligence (low) } \\
\text { (ii) Insecure } \\
\text { attachment } \\
\text { (iii) Peer } \\
\text { relationships }\end{array}$} & No & CWP & Population survey & Population & 6,902 & Yes & Gale et al. [51] & +++ \\
\hline & Possibly & CWP & $\begin{array}{c}\text { Prospective } \\
\text { population cohort }\end{array}$ & Population & 2,509 & No & Davies et al. [52] & +++ \\
\hline & Yes & FMS & Case-control & Clinic & 110 & No & $\begin{array}{l}\text { Kashikar-Zuck } \\
\quad \text { et al. [53] }\end{array}$ & + \\
\hline & Yes & CWP & $\begin{array}{c}\text { Prospective } \\
\text { population cohort }\end{array}$ & Population & 7,571 & Yes & $\begin{array}{l}\text { Jones et al. } \\
{[19,45,46]}\end{array}$ & +++ \\
\hline & & CWP & $\begin{array}{l}\text { Prospective } \\
\text { population cohort }\end{array}$ & Population & 7,470 & Yes & $\begin{array}{l}\text { Jones et al. } \\
{[19,45,46]}\end{array}$ & +++ \\
\hline & & CWP & $\begin{array}{l}\text { Prospective } \\
\text { population cohort }\end{array}$ & Population & 1,440 & Yes & $\begin{array}{l}\text { Jones et al. } \\
{[19,45,46]}\end{array}$ & +++ \\
\hline \multirow[t]{5}{*}{$\begin{array}{l}\text { (iv) Stress, anxiety, } \\
\text { and depression }\end{array}$} & & CWP & $\begin{array}{l}\text { Prospective } \\
\text { population cohort }\end{array}$ & Population & 2,650 & Yes & $\begin{array}{l}\text { Nicholl et al. } \\
\text { [54] }\end{array}$ & +++ \\
\hline & & CWP & Cross-sectional & Population & 7,070 & No & Skrove et al. [21] & ++ \\
\hline & & FMS & Population survey & Population & 3,006 & Yes & Wolfe et al. [55] & ++ \\
\hline & & FMS & Case series & Clinic & 76 & No & $\begin{array}{c}\text { Kashikar-Zuck } \\
\text { et al. [56] }\end{array}$ & + \\
\hline & & FMS & $\begin{array}{l}\text { Prospective } \\
\text { cohort }\end{array}$ & Clinic & 121 & Yes & $\begin{array}{l}\text { Cunningham } \\
\text { et al. [57] }\end{array}$ & +++ \\
\hline \multirow{3}{*}{$\begin{array}{l}\text { (v) Temperament } \\
\text { (vi) Posttraumatic } \\
\text { stress disorder } \\
\text { (vii) Socioeconomic } \\
\text { status (low) }\end{array}$} & Possibly & FMS & Case-control & Clinic & 48 & No & Conte et al. [58] & + \\
\hline & Possibly & CWP & $\begin{array}{c}\text { Cross-sectional } \\
\text { survey }\end{array}$ & Population & 3,740 & No & $\begin{array}{c}\text { Arguelles et al. } \\
\text { [59] }\end{array}$ & ++ \\
\hline & Possibly & CWP & $\begin{array}{c}\text { Prospective } \\
\text { population cohort }\end{array}$ & Population & 9,377 & Yes & $\begin{array}{c}\text { Macfarlane et al. } \\
{[60]}\end{array}$ & +++ \\
\hline Sleep problems & Yes & FMS & Case series & Clinic & 30 & No & $\begin{array}{c}\text { Tayag-Kier et al. } \\
{[61]}\end{array}$ & + \\
\hline Physical trauma & No & CWP & $\begin{array}{c}\text { Prospective } \\
\text { population cohort }\end{array}$ & Population & 7,571 & Yes & Jones et al. [45] & +++ \\
\hline
\end{tabular}


TABle 3: Continued.

\begin{tabular}{lcccccccc}
\hline Risk marker & Modifiable & $\begin{array}{c}\text { Pain } \\
\text { context }\end{array}$ & Type of study & $\begin{array}{c}\text { Population } \\
\text { or clinic }\end{array}$ & $\begin{array}{c}\text { Number of } \\
\text { participants }\end{array}$ & $\begin{array}{c}\text { Multivariate } \\
\text { analysis }\end{array}$ & $\begin{array}{c}\text { Reference } \\
\text { quality* }\end{array}$ \\
\hline $\begin{array}{l}\text { Functional/primary } \\
\text { pain disorders }\end{array}$ & Yes & CWP & $\begin{array}{c}\text { Prospective } \\
\text { population cohort } \\
\text { Prospective }\end{array}$ & Population & 7,470 & No & $\begin{array}{c}\text { Jones et al. } \\
{[19,46]}\end{array}$ & $\begin{array}{c}\text { Overall } \\
+++ \\
\text { Jones et al. } \\
{[19,46]}\end{array}$ \\
$\begin{array}{l}\text { Hypermobility of } \\
\text { joints }\end{array}$ & No & FMS & $\begin{array}{c}\text { Cross-sectional } \\
+++\end{array}$ & Clinic & 131 & No & Ting et al. [62] \\
$\begin{array}{l}\text { Chronic fatigue } \\
\begin{array}{l}\text { Small-fiber } \\
\text { polyneuropathy }\end{array}\end{array}$ & No & CWP & $\begin{array}{c}\text { Population birth } \\
\text { cohort }\end{array}$ & Population & 3214 & Yes & Norris et al. [63] ++++ \\
+
\end{tabular}

*Overall quality: + , very low; ++ , low; +++ , moderate; ++++ , high.

TABLE 4: Risk markers for chronic widespread pain and fibromyalgia in adult samples.

\begin{tabular}{|c|c|c|c|c|c|c|c|c|}
\hline Risk marker & Modifiable & $\begin{array}{c}\text { Pain } \\
\text { context }\end{array}$ & Type of study & $\begin{array}{l}\text { Population } \\
\text { or clinic }\end{array}$ & $\begin{array}{l}\text { Number of } \\
\text { participants }\end{array}$ & $\begin{array}{c}\text { Multivariate } \\
\text { analysis }\end{array}$ & Reference & $\begin{array}{l}\text { Overall } \\
\text { quality* }\end{array}$ \\
\hline \multirow{4}{*}{ Female sex } & \multirow[t]{4}{*}{ No } & CWP & $\begin{array}{c}\text { Prospective } \\
\text { population cohort }\end{array}$ & Population & 28,367 & Yes & $\begin{array}{l}\text { Mundal et al. } \\
{[66]}\end{array}$ & +++ \\
\hline & & CWP & Systematic review & $\mathrm{N} / \mathrm{A}$ & $\mathrm{N} / \mathrm{A}$ & $\mathrm{N} / \mathrm{A}$ & $\begin{array}{c}\text { Mansfield et al. } \\
\text { [67] }\end{array}$ & $\mathrm{N} / \mathrm{A}$ \\
\hline & & CWP & Review & $\mathrm{N} / \mathrm{A}$ & $\mathrm{N} / \mathrm{A}$ & N/A & Mogil [68] & N/A \\
\hline & & FMS & $\begin{array}{c}\text { Random } \\
\text { population survey }\end{array}$ & Population & 830 & No & $\begin{array}{l}\text { Vincent et al. } \\
\text { [69] }\end{array}$ & ++ \\
\hline \multicolumn{9}{|l|}{ Psychosocial factors } \\
\hline \multirow{3}{*}{$\begin{array}{l}\text { (i) Stress, anxiety, } \\
\text { and depression }\end{array}$} & \multirow[t]{3}{*}{ Yes } & CWP & $\begin{array}{c}\text { Prospective } \\
\text { population cohort }\end{array}$ & Population & 28,367 & Yes & $\begin{array}{l}\text { Mundal et al. } \\
{[66]}\end{array}$ & +++ \\
\hline & & CWP & $\begin{array}{l}\text { Prospective } \\
\text { population cohort }\end{array}$ & Population & 3,171 & Yes & Gupta et al. [70] & +++ \\
\hline & & CWP & Twin survey & $\begin{array}{l}\text { Population } \\
\text { Population } \\
\text { Population }\end{array}$ & $\begin{array}{c}3,266 \\
9,26 \\
3,266\end{array}$ & $\begin{array}{l}\text { Yes } \\
\text { Yes } \\
\text { Yes }\end{array}$ & $\begin{array}{l}\text { Burri et al. } \\
{[38,71,72]}\end{array}$ & ++++ \\
\hline $\begin{array}{l}\text { (ii) Emotional } \\
\text { intelligence (low) }\end{array}$ & Possibly & CWP & Twin survey & Population & 3,266 & Yes & Burri et al. [71] & ++++ \\
\hline \multirow{2}{*}{$\begin{array}{l}\text { (iii) Posttraumatic } \\
\text { stress disorder }\end{array}$} & \multirow[t]{2}{*}{ Possibly } & CWP & $\begin{array}{c}\text { Prospective } \\
\text { population cohort }\end{array}$ & Population & 1,312 & Yes & $\begin{array}{c}\text { Raphael et al. } \\
{[73]}\end{array}$ & +++ \\
\hline & & FMS & Cross-sectional & Clinic & 395 & No & $\begin{array}{c}\text { Häuser et al. } \\
{[74]}\end{array}$ & ++ \\
\hline \multirow{3}{*}{$\begin{array}{l}\text { (iv) Socioeconomic } \\
\text { status (low) } \\
\text { (v) Family } \\
\text { dysfunction } \\
\text { (vi) Maladjusted } \\
\text { behavior }\end{array}$} & Possibly & CWP & \multirow{3}{*}{$\begin{array}{l}\text { Prospective } \\
\text { population cohort } \\
\text { Case-control } \\
\text { cross-sectional } \\
\text { Prospective } \\
\text { population cohort }\end{array}$} & Population & 2,509 & No & $\begin{array}{c}\text { Davies et al. } \\
{[52]}\end{array}$ & +++ \\
\hline & Possible & CWP & & Clinic & 75 & Yes & $\begin{array}{c}\text { Hayaki et al. } \\
{[75]}\end{array}$ & ++ \\
\hline & Yes & CWP & & Population & 8572 & Yes & Pang et al. [76] & +++ \\
\hline Preterm birth & No & CWP & $\begin{array}{c}\text { Prospective } \\
\text { population cohort }\end{array}$ & Population & 8572 & Yes & $\begin{array}{c}\text { Littlejohn et al. } \\
\text { [77] }\end{array}$ & +++ \\
\hline \multirow[t]{2}{*}{ Sleep problems } & \multirow[t]{2}{*}{ Yes } & CWP & \multirow{2}{*}{$\begin{array}{c}\text { Prospective } \\
\text { population cohort } \\
\text { Review }\end{array}$} & Population & 3,171 & Yes & Gupta et al. [70] & +++ \\
\hline & & FMS & & $\mathrm{N} / \mathrm{A}$ & N/A & $\mathrm{N} / \mathrm{A}$ & Choy [78] & $\mathrm{N} / \mathrm{A}$ \\
\hline Physical trauma & No & CWP & $\begin{array}{c}\text { Prospective } \\
\text { cohort }\end{array}$ & Clinic & 948 & No & Hu et al. [79] & ++ \\
\hline \multirow{5}{*}{$\begin{array}{l}\text { Functional/primary } \\
\text { pain disorders }\end{array}$} & \multirow[t]{5}{*}{ Yes } & CWP & $\begin{array}{c}\text { Prospective } \\
\text { cohort }\end{array}$ & Clinic & 423 & Yes & Viniol et al. [80] & ++ \\
\hline & & CWP & $\begin{array}{l}\text { Retrospective } \\
\text { cohort }\end{array}$ & Clinic & 512 & Yes & $\begin{array}{c}\text { Kindler et al. } \\
{[81]}\end{array}$ & ++ \\
\hline & & FMS & Case-control & Clinic & 625 & Yes & $\begin{array}{c}\text { Warren et al. } \\
{[82]}\end{array}$ & ++ \\
\hline & & FMS & Cross-sectional & Clinic & 1,123 & Yes & $\begin{array}{l}\text { de Tommaso } \\
\text { et al. [83] }\end{array}$ & ++ \\
\hline & & FMS & Controlled trial & Clinic & 86 & No & $\begin{array}{c}\text { Giamberardino } \\
\text { et al. }[84]\end{array}$ & + \\
\hline
\end{tabular}


TABle 4: Continued.

\begin{tabular}{|c|c|c|c|c|c|c|c|c|}
\hline Risk marker & Modifiable & $\begin{array}{c}\text { Pain } \\
\text { context }\end{array}$ & Type of study & $\begin{array}{l}\text { Population } \\
\text { or clinic }\end{array}$ & $\begin{array}{l}\text { Number of } \\
\text { participants }\end{array}$ & $\begin{array}{c}\text { Multivariate } \\
\text { analysis }\end{array}$ & Reference & $\begin{array}{r}\text { Overall } \\
\text { quality* }\end{array}$ \\
\hline \multirow{3}{*}{$\begin{array}{l}\text { Overweight and } \\
\text { obesity }\end{array}$} & \multirow[t]{3}{*}{ Yes } & CWP & \multirow{3}{*}{$\begin{array}{c}\text { Prospective } \\
\text { population cohort } \\
\text { Prospective } \\
\text { population cohort } \\
\text { Review }\end{array}$} & Population & 28,367 & Yes & $\begin{array}{l}\text { Mundal et al. } \\
{[66]}\end{array}$ & +++ \\
\hline & & CWP & & Population & 1,553 & Yes & $\begin{array}{c}\text { Magnusson } \\
\text { et al. [85] }\end{array}$ & +++ \\
\hline & & FMS & & N/A & N/A & N/A & Ursini et al. [86] & N/A \\
\hline Iron deficiency & Yes & FMS & Case-control & Clinic & 92 & No & $\begin{array}{l}\text { Ortancil et al. } \\
\text { [87] }\end{array}$ & + \\
\hline Hemochromatosis & Yes & FMS & Cross-sectional & Clinic & 295 & No & $\begin{array}{c}\text { Mohammad } \\
\text { et al. [88] }\end{array}$ & + \\
\hline HPA dysfunction & Possibly & CWP & $\begin{array}{c}\text { Prospective } \\
\text { population cohort }\end{array}$ & Population & 241 & Yes & $\begin{array}{c}\text { McBeth et al. } \\
{[89]}\end{array}$ & +++ \\
\hline \multirow{3}{*}{ Infectious illness } & \multirow[t]{3}{*}{ Possibly } & FMS & Review & $\mathrm{N} / \mathrm{A}$ & $\mathrm{N} / \mathrm{A}$ & $\mathrm{N} / \mathrm{A}$ & $\begin{array}{c}\text { Buskila et al. } \\
{[90]}\end{array}$ & N/A \\
\hline & & FMS & Review & $\mathrm{N} / \mathrm{A}$ & N/A & N/A & $\begin{array}{c}\text { Häuser et al. } \\
{[91]}\end{array}$ & N/A \\
\hline & & FMS & $\begin{array}{c}\text { Prospective } \\
\text { cohort controlled }\end{array}$ & Population & 1,244 & Yes & Chen et al. [92] & +++ \\
\hline
\end{tabular}

*Overall quality: +, very low; ++, low; +++, moderate; ++++, high.

Reviewing eight twin studies on CWP, six molecular genetic studies on CWP, and one epigenetic study, the findings by Kerr and Burri [42] suggested that genetic and unique environmental factors contributed to CWP. Various candidate genes, such as serotonin-related pathway genes, were found to be associated with CWP.

Most studies which have documented psychosocial determinants of CWP have been unable to exclude genetic confounding, given the strong heritability of some risk markers, notably depression and anxiety. Burri et al. [71] used a discordant monozygotic-twin approach to overcome the traditional limitations of conventional epidemiology by separating environmental influences from genetic confounders to provide clues on the direction of causality. The results, supported by another twin study [38], demonstrated that the covariance between CWP and depression and anxiety was due to a common, highly heritable, and latent trait.

Research on genetic predisposition to FMS has revealed associations between genetic factors and FMS, including specific gene polymorphisms involved in serotonergic, dopaminergic, and catecholaminergic pathways [44, 94]. FMS coaggregated in families with reduced pressure pain thresholds and major mood disorder [43].

3.2. Female Sex. CWP and FMS demonstrated female predominance [95], with juvenile FMS affecting females four times more than males [29]. This female predominance is incompletely understood, but likely involves genetic, psychological, behavioral (including reporting behavior), and neurobiological factors [68].

3.3. Early-Life Adversity. Early-life adversity was a risk marker for developing adult CWP and FMS. Epidemiological studies of the 1958 British Birth Cohort Study demonstrated that multiple somatic symptoms at age 7 were independently associated with CWP at age $45[19,45,46]$. Moderate associations included headaches, abdominal pain, and periodic vomiting [46], and significant associations included hospitalization following a road traffic accident, residence in institutional care, maternal death, familial financial hardship, and persistent behavioral problems $[45,76,96]$. Whilst the association between preterm birth and very low birthweight and adult CWP was not statistically significant [77], extremely premature babies who underwent repeated procedures after birth demonstrated sustained changes in sensory processing by age 11 [97].

Other studies have demonstrated that painful infant experiences and childhood maltreatment were associated with adult FMS. These associations included preterm birth, perinatal exposure to substances of abuse, maternal deprivation, growing up with a depressed parent, psychological trauma, physical or verbal abuse from parents, and physical or sexual abuse by an adult [47-49]. Abuse in childhood was associated with a $97 \%$ increased risk of painful somatic syndromes in adulthood, including FMS [98].

Early-life adversity may increase vulnerability to CWP and FMS by excessively activating stress responses during a critical period and altering normal development, stress reactivity, and nociceptive function through hyperalgesic priming. The pain itself is a stressor and, as it evolves, may generate a positive feedback loop to increase anxiety levels and impact stress regulation.

3.4. Cognitive and Psychosocial Factors. Early childhood cognitive and psychosocial problems are risk markers for CWP. In children, the independent risk markers for widespread pain were adverse behavioral and emotional factors, somatic pain symptoms, older age, female sex, depression, and regional back and neck pain symptoms $[19,20]$. In adolescents, CWP was associated with anxiety, 
depression, and conduct or attentional problems [21]. For young people aged 10-18, higher widespread pain scores were associated with poorer mental and physical healthrelated quality of life and greater functional impairment. Whilst this relationship was explained by psychosocial distress, including somatization features, health-seeking behavior, insecure attachment style, and poor sleep, the causal direction has not been established [52, 54, 70, 99]. Resilience factors, including high self-esteem, seldom feeling lonely, and high scores for family cohesion or social confidence, were associated with a lower prevalence, and markedly attenuated the association between psychiatric symptoms and CWP [21].

In adults, prospective studies from the 1958 British Birth Cohort Study demonstrated that low socioeconomic status at age 7 was associated with CWP at age 45 [60] and appeared to be explained by psychological factors [52]. Additionally, low intellectual and emotional intelligence, low social class, low educational attainment, high body mass index, and psychological distress in childhood were independent predictors of CWP in adulthood [51].

In later life, risk markers for CWP included the psychological factors of anxiety and depression, lifestyle factors of smoking and obesity, and other factors such as dysfunction of the hypothalamic-pituitary-adrenal axis $[66,89]$. Posttraumatic stress disorder was strongly associated with CWP and FMS [59, 73, 74], likely mediated in part by the known enhanced influence on central sensitization and common antecedent traumatic experiences.

Juvenile FMS was associated with temperamental instability, low mood, depression and anxiety, family disorder, social isolation, higher pain and emotional sensitivity, and lower psychological adjustment $[53,58]$. Psychiatric disorders, notably anxiety, were prevalent in juvenile and early adulthood FMS and were associated with greater physical impairment, poorer functioning, and lower health-related quality of life $[56,57]$. The lifetime prevalence of psychiatric comorbidity, notably depression, anxiety, and bipolar disorder, with FMS may suggest a shared underlying pathophysiological link.

3.5. Sleep Problems. Poor self-reported sleep quality was a risk marker for CWP and FMS [78]. Whilst children with juvenile FMS demonstrated abnormal $\alpha$-rhythms (suggestive of wakefulness during nonrapid eye movement sleep), periodic limb movements, prolonged sleep latency, shortened total sleep time, decreased sleep efficiency, and increased wakefulness during sleep, the causal relationship is unclear [61]. Sleep deprivation impairs descending paininhibition pathways and can cause myalgia, tenderness, and fatigue, suggesting that sleep dysfunction might not only be a consequence of pain but also pathogenetic [78].

3.6. Physical Trauma. The 1958 British Birth Cohort Study demonstrated the association between childhood trauma and CWP [45]. One of the major childhood risk markers for adult CWP was hospitalization following a road traffic accident, though this may be influenced by the associated psychological sequalae.

Of individuals who presented to an emergency department after motor vehicle collision, $11 \%$ developed CWP [79]. This is consistent with evidence that spinal pain not uncommonly evolves into CWP. In adult females with FMS, childhood trauma had a clinically important association with loss of functionality and comorbid depression [100].

3.7. Functional/Primary Pain Disorders and Multiregional Pain Disorders. CWP and FMS often evolved over time from multiregional pain disorders. Headaches and abdominal pain in children were associated with a doubling of the risk of developing widespread body pain in the ensuing year [19]. Children aged 7 with abdominal pain, headaches, migraine, or vomiting attacks had an increased risk of CWP at age 45 [46].

Adults with back or neck pain were particularly at risk of CWP, and the same risk was likely to apply to children and adolescents [15]. The incidence of CWP in adults with chronic low back pain was $23.8 \%$ [80], and the rate of developing CWP within 5-6 years in adults with regional back or neck pain was $22.6 \%$ [81]. The risk markers associated with the transition to CWP were moderate or severe pain intensity, female sex, long duration of back pain, high rate of psychosomatic symptoms, history of abuse, family history of CWP, severe interference with general activities, having one or more central sensitivity syndromes, and using more pain management strategies. Additionally, functional somatic syndromes were risk markers for CWP, specifically chronic fatigue, irritable bowel syndrome, and chronic pelvic pain [82], and for FMS, specifically migraine [83, 84].

3.8. Hypermobility of Joints. In adolescents with juvenile FMS, $48 \%$ have been clinically assessed as having hypermobility of joints [62]. Hypermobility of joints was associated with enhanced physiological pain sensitivity.

3.9. Obesity. Obesity and low physical activity have been epidemiologically associated with chronic pain, including CWP $[66,86]$ and FMS $[86,101]$; however, studies in adolescents have only reported associations between obesity and the prevalence and severity of knee pain and chronic regional pain [102]. Obesity can be considered an aggravating comorbid condition affecting condition severity, quality of life, fatigue, and physical dysfunction. Poor physical fitness was a key covariate in increasing the risk of both widespread pain and obesity [85]. Other potential causal influences of obesity on CWP and FMS included physical inactivity, sleep disturbance, depression, endocrine dysfunction, lower pressure pain thresholds, and inflammatory and immune effects [103-105].

3.10. Rheumatic Conditions. The 2016 revised fibromyalgia criteria permitted a diagnosis of FMS in association with any medical illness, including inflammatory musculoskeletal disorders. There has been a tendency for CWP and a deep 
hyperalgesic state occurring in $10-30 \%$ of patients with primary rheumatic diseases, including rheumatoid arthritis and systemic lupus erythematosus, to be termed secondary or comorbid FMS. However, this is best referred to by the primary diagnosis and the additional interpretation of widespread deep secondary allodynia or low-pressure pain threshold reflecting central sensitization [95]. It is acknowledged that the inflammatory rheumatic condition might not be the sole source of nociceptive input or source of central somatosensory dysfunction.

3.11. Iron Deficiency. Iron deficiency, especially in early life, may be a risk marker for CWP. In a twin family study, we observed an independent association between iron deficiency and chronic pediatric pain [106]. A serum ferritin level $<50 \mathrm{ng} / \mathrm{ml}$ was associated with a 6.5 -fold increased risk for FMS in females [87]. Paradoxically, FMS has also been associated with hemochromatosis [88].

3.12. Infectious Illness. Various infections have been linked to FMS. Epstein-Barr and parvovirus infections, brucellosis, and Lyme disease were often cited [90,91], but any infectious illness characterized by prolonged bed rest and persistent fatigue might be a causal influence. Postinfection chronic fatigue syndrome is probably a more appropriate diagnostic term, especially in those in whom fatigue is dominant over pain. Microglial activation may underpin postinfectious contributions to CWP.

3.13. Small-FiberPolyneuropathy. Small-fiber polyneuropathy, alongside chronic neuroinflammation, was associated with CWP and FMS, with claims of causation. In patients with widespread pain beginning before age 21, small-fiber polyneuropathy was "definite" in 59\% and "probable" in 17\% [64].

\section{Discussion}

The literature leaves little doubt that some children and adolescents have increased vulnerability to developing CWP and FMS. The risk markers for CWP and FMS suggest a complex etiological structure which shares risk markers with those identified in other chronic pain disorders. Evidence for potential mechanisms underpinning these risk markers is emerging at molecular, cellular, and network levels [107]. Alterations in the structure, wiring, function, and neurochemistry of various brain networks, particularly the reward-motivation network and the endogenous pain modulation system, might be involved in conferring vulnerability to pain conditions. Epigenetic modulation, leading to changes to neuronal and molecular processes, is one way in which genetics and adverse priming events, including prior injury or stressful environmental influences, may increase risk. In FMS, neuropathological changes include regional changes in grey matter volume, decreased functional connectivity in the descending pain-modulating system, and an increased activity in the pain matrix related to central sensitization. Whether measurable alterations in brain function precede or follow the onset of chronic pain, they might lead to a vicious cycle in which vulnerability leads to nonresilience to additional factors arising from the chronic pain state. It is likely that biological, psychological, social, and contextual risk markers interact to predispose, initiate, maintain, and exacerbate CWP and FMS [72].

A model of life-course influences on the development of chronic pain was published by Dominick and Blythe [108]. The model by Von Baeyer and Champion [12] was developed to illustrate factors in the development of multiple functional (or primary) pain disorders, but could appropriately be applied to life-course risk markers in the development of CWP and FMS in young people. It is likely that biological, psychological, social, and contextual variables interact to influence the predisposition, triggering, and aggravation of CWP and FMS. To integrate current knowledge, theory, and hypotheses, we propose a biopsychosocial model of risk markers for chronic widespread pain and fibromyalgia syndrome in young people (Figure 1). For a more specific model for FMS, see Häuser et al. [91], and for juvenile FMS, see Kashikar-Zuck and Ting [28].

The majority of the risk markers have at least some potential for modification. There is less or no prospect of favorable modification of sex, temperament, intelligence, hypermobility of joints, small-fiber polyneuropathy, physical trauma, and chronic fatigue.

Although this review was based on extensive literature review and evaluation of the strength of research evidence, the review was limited by the possible selectivity of searches and citations, and publication bias underestimating negative results.

\section{Conclusion}

The strongest evidence for risk markers as potential causal influences on CWP and FMS (Tables 2-4) came from prospective cohort studies, notably birth cohorts, and genetic studies, mainly twin analyses. Genetic influences were prominent but not always overt, including shared genes between widespread pain and depression, widespread pain and spinal pain, and functional/primary pain syndromes. Low emotional intelligence merits more attention, given the strength of evidence. The social risk markers, including childhood adversity and low socioeconomic status, were prominent across numerous studies and were at least partly related to stress, anxiety, and depression.

The case presented illustrates how a history of risk markers over the life-course can provide insight into why a young person might acquire CWP fulfilling criteria for FMS. Her risk markers were family history, premature birth, childhood adversity, hypermobility of joints, mild obesity, anxiety and depression, multiple primary or functional pain disorders, physical trauma, iron deficiency, and infectious illness with chronic fatigue. Such a cumulative burden evidently determined the onset by adolescence of her CWP, but typically the diagnostic categorization was made several years after the symptoms had become well established, 


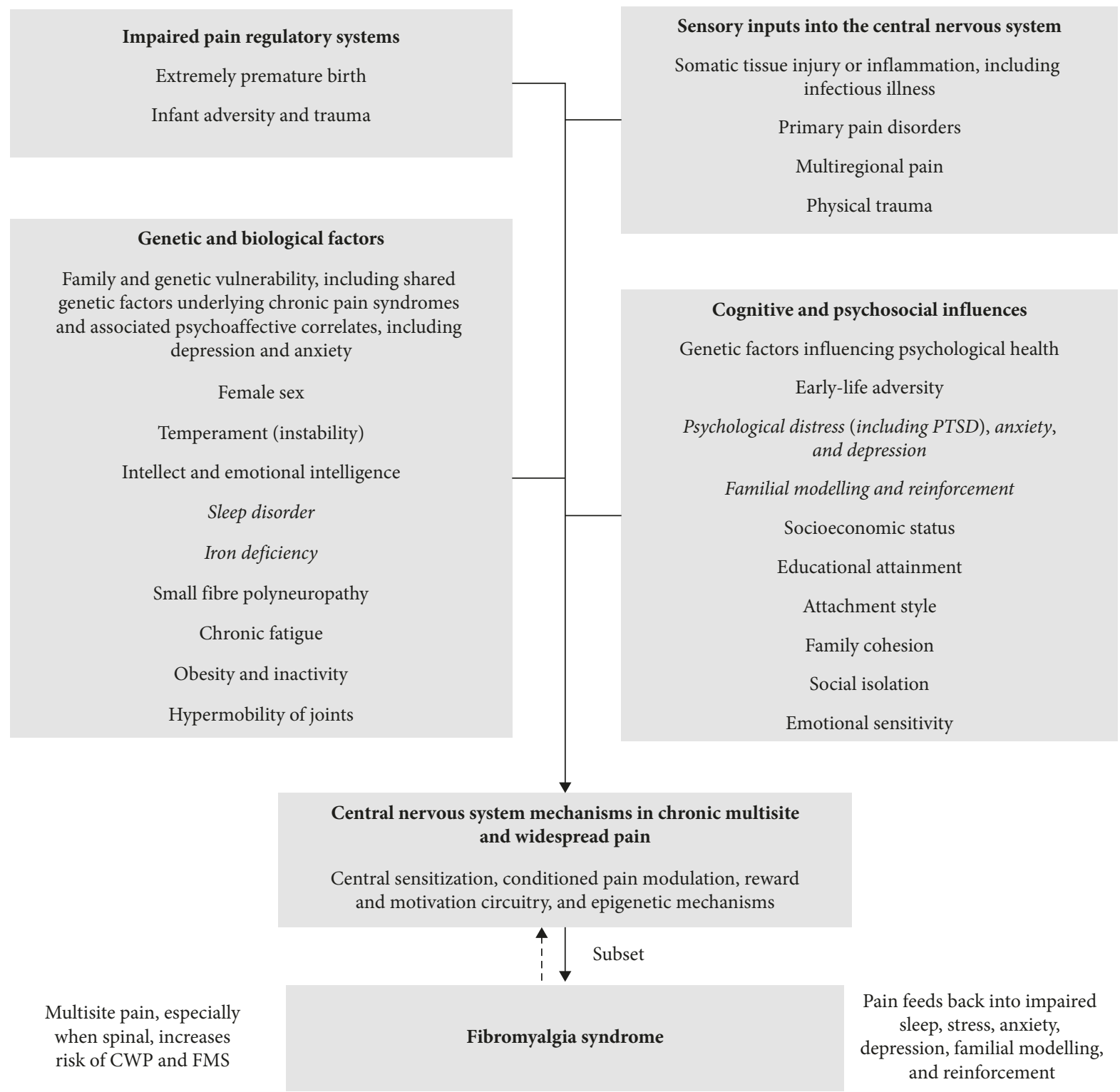

Figure 1: Biopsychosocial model of risk markers for chronic widespread pain and fibromyalgia syndrome in young people. Note. Risk markers for which there is limited evidence have been italicized.

notwithstanding consultations and intensive investigations. The taking of an earlier insightful history of the risk markers could have identified reversible factors leading to a more favorable trajectory and might have worked to mutual advantage for the patient and physician. It is likely that personal resilience can limit the severity of CWP and FMS and warrants further research with an aim to developing interventions designed to increase personal resilience.

\section{Abbreviations}

CWP: Chronic widespread pain

FMS: Fibromyalgia syndrome.

\section{Conflicts of Interest}

The authors report no conflicts of interest in this work.

\section{Acknowledgments}

The technical support of Theresa Donnelly and Ashton Curry-Hyde is gratefully acknowledged, as is the inspiration provided by the patient.

\section{References}

[1] M. López-Solà, C.-W. Woo, J. Pujol et al., "Towards a neurophysiological signature for fibromyalgia," Pain, vol. 158, no. 1, pp. 34-47, 2017.

[2] B. Gerdle, B. Ghafouri, N. Ghafouri, E. Backryd, and T. Gordh, "Signs of ongoing inflammation in female patients with chronic widespread pain: a multivariate, explorative, cross-sectional study of blood samples," Medicine, vol. 96, no. 9, article e6130, 2017.

[3] K. Wåhlén, P. Olausson, A. K. Carlsson, N. Ghafouri, B. Gerdle, and B. Ghafouri, "Systemic alterations in plasma 
proteins from women with chronic widespread pain compared to healthy controls: a proteomic study," Journal of Pain Research, vol. 10, pp. 797-809, 2017.

[4] B. A. Burt, "Defintions of risk," Journal of Dental Education, vol. 65, no. 10, pp. 1007-1008, 2001.

[5] D. P. Strachan, A. R. Rudnicka, C. Power et al., "Lifecourse influences on health among British adults: effects of region of residence in childhood and adulthood," International Journal of Epidemiology, vol. 36, no. 3, pp. 522-531, 2007.

[6] G. J. Macfarlane, Life-Course Influences on Chronic Pain in Adults, Oxford University Press, Vol. 1, Oxford, UK, 2010.

[7] J. Oster, "Recurrent abdominal pain, headache and limb pains in children and adolescents," Pediatrics, vol. 50, no. 3, pp. 429-436, 1972.

[8] J. Salminen, J. Pentti, and P. Terho, "Low back pain and disability in 14-year-old schoolchildren," Acta Paediatrica, vol. 81, no. 12, pp. 1035-1039, 1992.

[9] S. King, C. T. Chambers, A. Huguet et al., "The epidemiology of chronic pain in children and adolescents revisited: a systematic review," Pain, vol. 152, no. 12, pp. 2729-2738, 2011.

[10] B. Kroner-Herwig, J. Gassmann, H. van Gessel, and N. Vath, "Multiple pains in children and adolescents: a risk factor analysis in a longitudinal study," Journal of Pediatric Psychology, vol. 36, no. 4, pp. 420-432, 2011.

[11] C. W. Perquin, A. A. J. M. Hazebroek-Kampschreur, J. A. M. Hunfeld et al., "Pain in children and adolescents: a common experience," Pain, vol. 87, no. 1, pp. 51-58, 2000.

[12] C. L. von Baeyer and G. D. Champion, "Commentary: multiple pains as functional pain syndromes," Journal of Pediatric Psychology, vol. 36, no. 4, pp. 433-437, 2011.

[13] B. Natvig, C. Ihlebæk, Y. Kamaleri, and D. Bruusgaard, Number of Pain Sites-A Simple Measure of Population Risk?, Oxford University Press, Vol. 1, Oxford, UK, 2010.

[14] P. Croft, "The epidemiology of chronic widespread pain," Journal of Musculoskeletal Pain, vol. 10, no. 1-2, pp. 191-199, 2002.

[15] K. D. Watson, A. C. Papageorgiou, G. T. Jones et al., "Low back pain in schoolchildren: the role of mechanical and psychosocial factors," Archives of Disease in Childhood, vol. 88, no. 1, pp. 12-17, 2003.

[16] A. Coenders, C. Chapman, P. Hannaford et al., "In search of risk factors for chronic pain in adolescents: a case-control study of childhood and parental associations," Journal of Pain Research, vol. 7, pp. 175-183, 2014.

[17] A. L. Holley, A. C. Wilson, and T. M. Palermo, "Predictors of the transition from acute to persistent musculoskeletal pain in children and adolescents," Pain, vol. 158, no. 5, pp. 794-801, 2017.

[18] F. Wolfe, H. A. Smythe, M. B. Yunus et al., "The american college of rheumatology 1990 criteria for the classification of fibromyalgia," Arthritis \& Rheumatism, vol. 33, no. 2, pp. 160-172, 1990.

[19] G. T. Jones, A. J. Silman, and G. J. Macfarlane, "Predicting the onset of widespread body pain among children," Arthritis \& Rheumatism, vol. 48, no. 9, pp. 2615-2621, 2003.

[20] M. Mikkelsson, A. El-Metwally, H. Kautiainen, A. Auvinen, G. J. Macfarlane, and J. J. Salminen, "Onset, prognosis and risk factors for widespread pain in schoolchildren: a prospective 4-year follow-up study," Pain, vol. 138, no. 3, pp. 681-687, 2008.

[21] M. Skrove, P. Romundstad, and M. S. Indredavik, "Chronic multisite pain in adolescent girls and boys with emotional and behavioral problems: the Young-HUNT study,"
European Child \& Adolescent Psychiatry, vol. 24, no. 5, pp. 503-515, 2015.

[22] S. J. Hoogwout, M. V. Paananen, A. J. Smith et al., "Musculoskeletal pain is associated with restless legs syndrome in young adults," BMC Musculoskeletal Disorder, vol. 16, no. 1, p. 294, 2015.

[23] D. J. Clauw, L. M. Arnold, and B. H. McCarberg, "The science of fibromyalgia," Mayo Clinic Proceedings, vol. 86, no. 9, pp. 907-911, 2011.

[24] E. Bäckryd, L. Tanum, A.-L. Lind, A. Larsson, and T. Gordh, "Evidence of both systemic inflammation and neuroinflammation in fibromyalgia patients, as assessed by a multiplex protein panel applied to the cerebrospinal fluid and to plasma," Journal of Pain Research, vol. 10, pp. 515$525,2017$.

[25] F. Wolfe, D. J. Clauw, M.-A. Fitzcharles et al., "2016 revisions to the 2010/2011 fibromyalgia diagnostic criteria," Seminars in Arthritis and Rheumatism, vol. 46, no. 3, pp. 319-329, 2016.

[26] F. Wolfe, D. J. Clauw, M.-A. Fitzcharles et al., "The American college of rheumatology preliminary diagnostic criteria for fibromyalgia and measurement of symptom severity," Arthritis Care \& Research, vol. 62, no. 5, pp. 600-610, 2010.

[27] F. Wolfe, "How to use the new American College of Rheumatology fibromyalgia diagnostic criteria," Arthritis Care \& Research, vol. 63, no. 7, pp. 1073-1074, 2011.

[28] S. Kashikar-Zuck and T. V. Ting, "Juvenile fibromyalgia: current status of research and future developments," Nature Reviews Rheumatology, vol. 10, no. 2, pp. 89-96, 2014.

[29] S. Gmuca and D. D. Sherry, "Fibromyalgia: treating pain in the juvenile patient," Pediatric Drugs, vol. 19, no. 4, pp. 325-338, 2017.

[30] C. M. Hoffart and D. D. Sherry, "Fibromyalgia-toward a definition in children," Journal of Pediatrics, vol. 169, pp. 9-10, 2016.

[31] T. V. Ting, K. Barnett, A. Lynch-Jordan, C. Whitacre, M. Henrickson, and S. Kashikar-Zuck, "2010 American College of Rheumatology adult fibromyalgia criteria for use in an adolescent female population with juvenile fibromyalgia," Journal of Pediatrics, vol. 169, pp. 181.e1-187.e1, 2016.

[32] Y. Kimura and G. A. Walco, Fibromyalgia in Children and Adolescents: Clinical Manifestations and Diagnosis, UpToDate, Waltham, MA, USA, 2016.

[33] S. Kashikar-Zuck, N. Cunningham, S. Sil et al., "Long-term outcomes of adolescents with juvenile-onset fibromyalgia in early adulthood," Pediatrics, vol. 133, no. 3, pp. e592-e600, 2014.

[34] M. Nicholas, J. W. S. Vlaeyen, W. Rief et al., "The IASP classification of chronic pain for ICD-11," Pain, vol. 160, no. 1, pp. 28-37, 2019.

[35] M. Aydede and A. Shriver, "Recently introduced definition of "nociplastic pain" by the International Association for the Study of Pain needs better formulation," Pain, vol. 159, no. 6, pp. 1176-1177, 2018.

[36] A. Huguet, J. A. Hayden, J. Stinson et al., "Judging the quality of evidence in reviews of prognostic factor research: adapting the GRADE framework," Systematic Reviews, vol. 2, no. 1, 2013.

[37] K. Kato, P. F. Sullivan, B. Evengård, and N. L. Pedersen, "Importance of genetic influences on chronic widespread pain," Arthritis \& Rheumatism, vol. 54, no. 5, pp. 1682-1686, 2006.

[38] A. Burri, S. Ogata, G. Livshits, and F. Williams, "The association between chronic widespread musculoskeletal pain, 
depression and fatigue is genetically mediated," PLoS One, vol. 10, no. 11, Article ID e0140289, 2015.

[39] J. Vehof, H. M. S. Zavos, G. Lachance, C. J. Hammond, and F. M. K. Williams, "Shared genetic factors underlie chronic pain syndromes," Pain, vol. 155, no. 8, pp. 1562-1568, 2014.

[40] I. Malkin, F. M. K. Williams, G. LaChance, T. Spector, A. J. MacGregor, and G. Livshits, "Low back and common widespread pain share common genetic determinants," Annals of Human Genetics, vol. 78, no. 5, pp. 357-366, 2014.

[41] J. R. Zadro, T. I. L. Nilsen, D. Shirley, A. B. Amorim, P. H. Ferreira, and P. J. Mork, "Parental chronic widespread pain and the association with chronic widespread pain in adult offspring: family-linkage data from the Norwegian HUNT Study," European Journal of Pain, vol. 22, no. 8, pp. 1485-1493, 2018.

[42] J. I. Kerr and A. Burri, "Genetic and epigenetic epidemiology of chronic widespread pain," Journal of Pain Research, vol. 10, pp. 2021-2029, 2017.

[43] L. M. Arnold, J. I. Hudson, E. V. Hess et al., "Family study of fibromyalgia," Arthritis \& Rheumatism, vol. 50, no. 3, pp. 944-952, 2004.

[44] D.-J. Park, J.-H. Kang, Y.-R. Yim et al., "Exploring genetic susceptibility to fibromyalgia," Chonnam Medical Journal, vol. 51, no. 2, pp. 58-65, 2015.

[45] G. T. Jones, C. Power, and G. J. Macfarlane, "Adverse events in childhood and chronic widespread pain in adult life: results from the 1958 British Birth Cohort Study," Pain, vol. 143, no. 1, pp. 92-96, 2009.

[46] G. T. Jones, A. J. Silman, C. Power, and G. J. Macfarlane, "Are common symptoms in childhood associated with chronic widespread body pain in adulthood?: results from the 1958 British birth cohort study," Arthritis \& Rheumatism, vol. 56, no. 5, pp. 1669-1675, 2007.

[47] P. Olivieri, B. Solitar, and M. Dubois, "Childhood risk factors for developing fibromyalgia," Open Access Rheumatology: Research and Reviews, vol. 109, 2012.

[48] D. Bohn, K. Bernardy, F. Wolfe, and W. Häuser, "The association among childhood maltreatment, somatic symptom intensity, depression, and somatoform dissociative symptoms in patients with fibromyalgia syndrome: a single-center cohort study," Journal of Trauma \& Dissociation, vol. 14, no. 3, pp. 342-358, 2013.

[49] L. A. Low and P. Schweinhardt, "Early life adversity as a risk factor for fibromyalgia in later life," Pain Research and Treatment, vol. 2012, Article ID 140832, 15 pages, 2012.

[50] R. Hellou, W. Hauser, I. Brenner et al., "Self-reported childhood maltreatment and traumatic events among Israeli patients suffering from fibromyalgia and rheumatoid arthritis," Pain Research and Management, vol. 2017, Article ID 3865249, 8 pages, 2017.

[51] C. R. Gale, I. J. Deary, C. Cooper, and D. G. Batty, "Intelligence in childhood and chronic widespread pain in middle age: The National Child Development Survey," Pain, vol. 153, no. 12, pp. 2339-2344, 2012.

[52] K. A. Davies, G. J. Macfarlane, J. McBeth, R. Morriss, and C. Dickens, "Insecure attachment style is associated with chronic widespread pain," Pain, vol. 143, no. 3, pp. 200-205, 2009.

[53] S. Kashikar-Zuck, A. M. Lynch, T. B. Graham, N. F. Swain, S. M. Mullen, and R. B. Noll, "Social functioning and peer relationships of adolescents with juvenile fibromyalgia syndrome," Arthritis \& Rheumatism, vol. 57, no. 3, pp. 474-480, 2007.
[54] B. I. Nicholl, G. J. Macfarlane, K. A. Davies, R. Morriss, C. Dickens, and J. McBeth, "Premorbid psychosocial factors are associated with poor health-related quality of life in subjects with new onset of chronic widespread pain-results from the EPIFUND study," Pain, vol. 141, no. 1, pp. 119-126, 2009.

[55] F. Wolfe, K. Ross, J. Anderson, I. J. Russell, and L. Hebert, "The prevalence and characteristics of fibromyalgia in the general population," Arthritis \& Rheumatism, vol. 38, no. 1, pp. 19-28, 1995.

[56] S. Kashikar-Zuck, I. S. Parkins, T. B. Graham et al., "Anxiety, mood, and behavioral disorders among pediatric patients with juvenile fibromyalgia syndrome," Clinical Journal of Pain, vol. 24, no. 7, pp. 620-626, 2008.

[57] N. R. Cunningham, S. T. Tran, A. M. Lynch-Jordan et al., "Psychiatric disorders in young adults diagnosed with juvenile fibromyalgia in adolescence," Journal of Rheumatology, vol. 42, no. 12, pp. 2427-2433, 2015.

[58] P. M. Conte, G. A. Walco, and Y. Kimura, "Temperament and stress response in children with juvenile primary fibromyalgia syndrome," Arthritis \& Rheumatism, vol. 48, no. 10, pp. 2923-2930, 2003.

[59] L. M. Arguelles, N. Afari, D. S. Buchwald, D. J. Clauw, S. Furner, and J. Goldberg, "A twin study of posttraumatic stress disorder symptoms and chronic widespread pain," Pain, vol. 124, no. 1, pp. 150-157, 2006.

[60] G. J. Macfarlane, G. Norrie, K. Atherton, C. Power, and G. T. Jones, "The influence of socioeconomic status on the reporting of regional and widespread musculoskeletal pain: results from the 1958 British Birth Cohort Study," Annals of the Rheumatic Diseases, vol. 68, no. 10, pp. 1591-1595, 2009.

[61] C. E. Tayag-Kier, G. F. Keenan, L. V. Scalzi et al., "Sleep and periodic limb movement in sleep in juvenile fibromyalgia," Pediatrics, vol. 106, no. 5, p. e70, 2000.

[62] T. V. Ting, P. J. Hashkes, K. Schikler, A. M. Desai, S. Spalding, and S. Kashikar-Zuck, "The role of benign joint hypermobility in the pain experience in Juvenile Fibromyalgia: an observational study," Pediatric Rheumatology, vol. 10, no. 1, p. 16, 2012.

[63] T. Norris, K. Deere, J. H. Tobias, and E. Crawley, "Chronic fatigue syndrome and chronic widespread pain in adolescence: population birth cohort study," Journal of Pain, vol. 18, no. 3, pp. 285-294, 2017.

[64] A. L. Oaklander and M. M. Klein, "Evidence of small-fiber polyneuropathy in unexplained, juvenile-onset, widespread pain syndromes," Pediatrics, vol. 131, no. 4, pp. e1091-e1100, 2013.

[65] H. Balshem, M. Helfand, H. J. Schünemann et al., "GRADE guidelines: 3. Rating the quality of evidence," Journal of Clinical Epidemiology, vol. 64, no. 4, pp. 401-406, 2011.

[66] I. Mundal, R. W. Grawe, J. H. Bjorngaard, O. M. Linaker, and E. A. Fors, "Prevalence and long-term predictors of persistent chronic widespread pain in the general population in an 11-year prospective study: the HUNT study," BMC Musculoskeletal Disorders, vol. 15, no. 1, p. 213, 2014.

[67] K. E. Mansfield, J. Sim, J. L. Jordan, and K. P. Jordan, “A systematic review and meta-analysis of the prevalence of chronic widespread pain in the general population," Pain, vol. 157, no. 1, pp. 55-64, 2016.

[68] J. S. Mogil, "Sex differences in pain and pain inhibition: multiple explanations of a controversial phenomenon," Nature Reviews Neuroscience, vol. 13, no. 12, pp. 859-866, 2012. 
[69] A. Vincent, B. D. Lahr, F. Wolfe et al., "Prevalence of fibromyalgia: a population-based study in olmsted county, Minnesota, utilizing the rochester epidemiology project," Arthritis Care \& Research, vol. 65, no. 5, pp. 786-792, 2013.

[70] A. Gupta, A. J. Silman, D. Ray et al., "The role of psychosocial factors in predicting the onset of chronic widespread pain: results from a prospective population-based study," Rheumatology, vol. 46, no. 4, pp. 666-671, 2006.

[71] A. Burri, G. Lachance, and F. Williams, "A discordant monozygotic-twin approach to potential risk factors for chronic widespread pain in females," Twin Research and Human Genetics, vol. 18, no. 2, pp. 188-197, 2015.

[72] A. Burri, S. Ogata, J. Vehof, and F. Williams, "Chronic widespread pain,” Pain, vol. 156, no. 8, pp. 1458-1464, 2015.

[73] K. G. Raphael, M. N. Janal, and S. Nayak, "Comorbidity of fibromyalgia and posttraumatic stress disorder symptoms in a community sample of women," Pain Medicine, vol. 5, no. 1, pp. 33-41, 2004.

[74] W. Häuser, A. Galek, B. Erbslöh-Möller et al., "Posttraumatic stress disorder in fibromyalgia syndrome: prevalence, temporal relationship between posttraumatic stress and fibromyalgia symptoms, and impact on clinical outcome," Pain, vol. 154, no. 8, pp. 1216-1223, 2013.

[75] C. Hayaki, K. Anno, M. Shibata et al., "Family dysfunction," Medicine, vol. 95, no. 49, article e5495, 2016.

[76] D. Pang, G. T. Jones, C. Power, and G. J. Macfarlane, "Influence of childhood behaviour on the reporting of chronic widespread pain in adulthood: results from the 1958 British Birth Cohort Study," Rheumatology, vol. 49, no. 10, pp. 1882-1888, 2010.

[77] C. Littlejohn, D. Pang, C. Power, G. J. Macfarlane, and G. T. Jones, "Is there an association between preterm birth or low birthweight and chronic widespread pain? Results from the 1958 Birth Cohort Study," European Journal of Pain, vol. 16, no. 1, pp. 134-139, 2012.

[78] E. H. S. Choy, "The role of sleep in pain and fibromyalgia," Nature Reviews Rheumatology, vol. 11, no. 9, pp. 513-520, 2015.

[79] J. Hu, A. V. Bortsov, L. Ballina et al., "Chronic widespread pain after motor vehicle collision typically occurs through immediate development and nonrecovery," Pain, vol. 157, no. 2, pp. 438-444, 2016.

[80] A. Viniol, N. Jegan, M. Brugger et al., "Even worse-risk factors and protective factors for transition from chronic localized low back pain to chronic widespread pain in general practice," Spine, vol. 40, no. 15, pp. E890-E899, 2015.

[81] L. L. Kindler, K. D. Jones, N. Perrin, and R. M. Bennett, "Risk factors predicting the development of widespread pain from chronic back or neck pain," Journal of Pain, vol. 11, no. 12, pp. 1320-1328, 2010.

[82] J. W. Warren, P. Langenberg, and D. J. Clauw, "The number of existing functional somatic syndromes (FSSs) is an important risk factor for new, different FSSs," Journal of Psychosomatic Research, vol. 74, no. 1, pp. 12-17, 2013.

[83] M. de Tommaso, A. Federici, C. Serpino et al., "Clinical features of headache patients with fibromyalgia comorbidity," Journal of Headache and Pain, vol. 12, no. 6, pp. 629638, 2011.

[84] M. A. Giamberardino, G. Affaitati, P. Martelletti et al., "Impact of migraine on fibromyalgia symptoms," Journal of Headache and Pain, vol. 17, no. 1, p. 28, 2016.

[85] K. Magnusson, K. B. Hagen, and B. Natvig, "Individual and joint effects of risk factors for onset widespread pain and obesity-a population-based prospective cohort study," European Journal of Pain, vol. 20, no. 7, pp. 1102-1110, 2016.

[86] F. Ursini, S. Naty, and R. D. Grembiale, "Fibromyalgia and obesity: the hidden link," Rheumatology International, vol. 31, no. 11, pp. 1403-1408, 2011.

[87] O. Ortancil, A. Sanli, R. Eryuksel, A. Basaran, and H. Ankarali, "Association between serum ferritin level and fibromyalgia syndrome," European Journal of Clinical Nutrition, vol. 64, no. 3, pp. 308-312, 2010.

[88] A. Mohammad, J. J. Carey, E. Storan, M. Scarry, R. J. Coughlan, and J. M. Lee, "High prevalence of fibromyalgia in patients with HFE-related hereditary hemochromatosis," Journal of Clinical Gastroenterology, vol. 47, no. 6, pp. 559-564, 2013.

[89] J. McBeth, A. J. Silman, A. Gupta et al., "Moderation of psychosocial risk factors through dysfunction of the hypothalamic-pituitary-adrenal stress axis in the onset of chronic widespread musculoskeletal pain: findings of a population-based prospective cohort study," Arthritis \& Rheumatism, vol. 56, no. 1, pp. 360-371, 2007.

[90] D. Buskila, F. Atzeni, and P. Sarzi-Puttini, "Etiology of fibromyalgia: the possible role of infection and vaccination," Autoimmunity Reviews, vol. 8, no. 1, pp. 41-43, 2008.

[91] W. Häuser, J. Ablin, M.-A. Fitzcharles et al., "Fibromyalgia," Nature Reviews Disease Primers, vol. 1, p. 15022, 2015.

[92] J.-H. Chen, C.-H. Muo, C.-H. Kao, C.-H. Tsai, and C.-H. Tseng, "Increased risk of new-onset fibromyalgia among chronic osteomyelitis patients: evidence from a taiwan cohort study," Journal of Pain, vol. 18, no. 2, pp. 222227, 2017.

[93] J. Vehof and F. M. Williams, "Genetic predisposition to chronic pain: from evolutionary advantage to a debilitating disease spectrum?," Pain Management, vol. 4, no. 6, pp. 381-383, 2014.

[94] T. A. Manolio, F. S. Collins, N. J. Cox et al., "Finding the missing heritability of complex diseases," Nature, vol. 461, no. 7265, pp. 747-753, 2009.

[95] D. J. Clauw, "Fibromyalgia," JAMA, vol. 311, no. 15, pp. 1547-1555, 2014.

[96] F. M. Blyth, G. T. Jones, and G. J. Macfarlane, "Musculoskeletal health-how early does it start?," Rheumatology, vol. 48, no. 10, pp. 1181-1182, 2009.

[97] S. M. Walker, L. S. Franck, M. Fitzgerald, J. Myles, J. Stocks, and N. Marlow, "Long-term impact of neonatal intensive care and surgery on somatosensory perception in children born extremely preterm," Pain, vol. 141, no. 1, pp. 79-87, 2009.

[98] R. R. Edwards, R. H. Dworkin, M. D. Sullivan, D. C. Turk, and A. D. Wasan, "The role of psychosocial processes in the development and maintenance of chronic pain," Journal of Pain, vol. 17, no. 9, pp. T70-T92, 2016.

[99] J. A. Rabbitts, A. L. Holley, C. B. Groenewald, and T. M. Palermo, "Association between widespread pain scores and functional impairment and health-related quality of life in clinical samples of children," Journal of Pain, vol. 17, no. 6, pp. 678-684, 2016.

[100] A. P. M. Filippon, D. G. Bassani, R. W. d. Aguiar, and L. H. F. Ceitlin, "Association between childhood trauma and loss of functionality in adult women with fibromyalgia," Trends in Psychiatry and Psychotherapy, vol. 35, no. 1, pp. 46-54, 2013.

[101] C. E. Gota, S. Kaouk, and W. S. Wilke, "Fibromyalgia and obesity," JCR: Journal of Clinical Rheumatology, vol. 21, no. 6, pp. 289-295, 2015. 
[102] K. C. Deere, J. Clinch, K. Holliday et al., "Obesity is a risk factor for musculoskeletal pain in adolescents: findings from a population-based cohort," Pain, vol. 153, no. 9, pp. 1932-1938, 2012.

[103] C. A. Paley and M. I. Johnson, "Physical activity to reduce systemic inflammation associated with chronic pain and obesity," Clinical Journal of Pain, vol. 32, no. 4, pp. 365-370, 2016.

[104] O. A. Tashani, R. Astita, D. Sharp, and M. I. Johnson, "Body mass index and distribution of body fat can influence sensory detection and pain sensitivity," European Journal of Pain, vol. 21, no. 7, pp. 1186-1196, 2017.

[105] F. W. Booth, C. K. Roberts, J. P. Thyfault, G. N. Ruegsegger, and R. G. Toedebusch, "Role of inactivity in chronic diseases: evolutionary insight and pathophysiological mechanisms," Physiological Reviews, vol. 97, no. 4, pp. 1351-1402, 2017.

[106] M. Crawford, H. Vo, M. Bui et al., "Parent-reported iron deficiency: heritability and associations with common pain disorders of childhood," in Proceedings of the World Congress on Pain, Buenos Aires, Argentina, November 2014.

[107] F. Denk, S. B. McMahon, and I. Tracey, "Pain vulnerability: a neurobiological perspective," Nature Neuroscience, vol. 17, no. 2, pp. 192-200, 2014.

[108] C. H. Dominick and F. M. Blyth, Epidemiology of Pain and Non-Pain Comorbidities, International Association for the Study of Pain, Washington, DC, USA, 1st edition, 2012. 


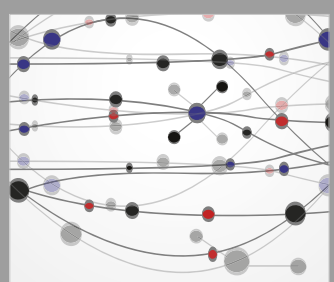

The Scientific World Journal
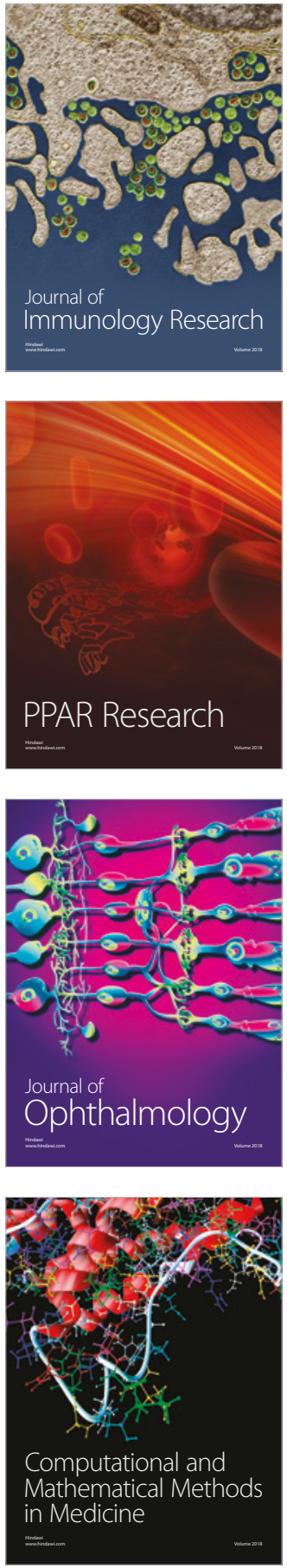

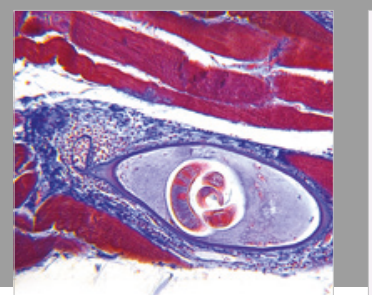

Gastroenterology Research and Practice

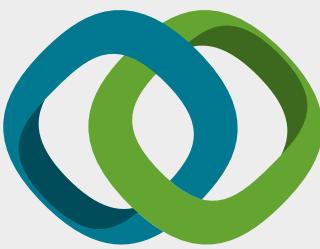

\section{Hindawi}

Submit your manuscripts at

www.hindawi.com
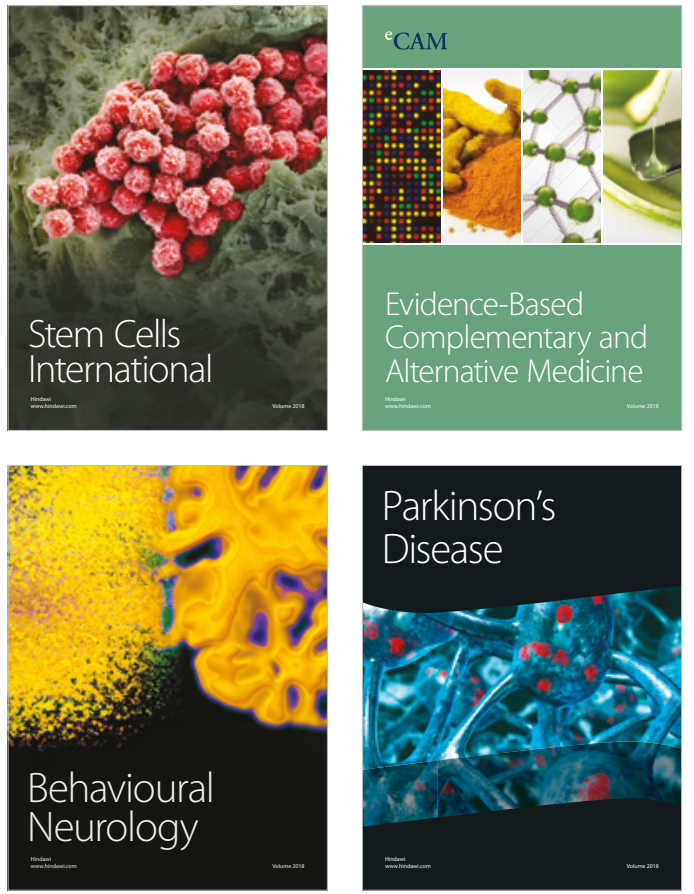

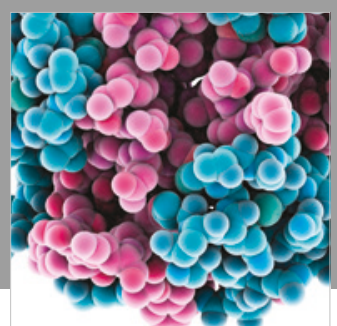

ournal of

Diabetes Research

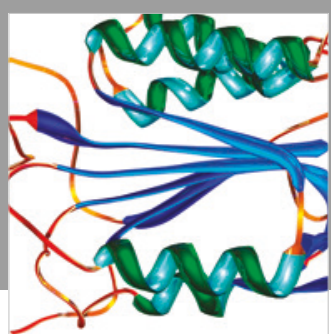

Disease Markers
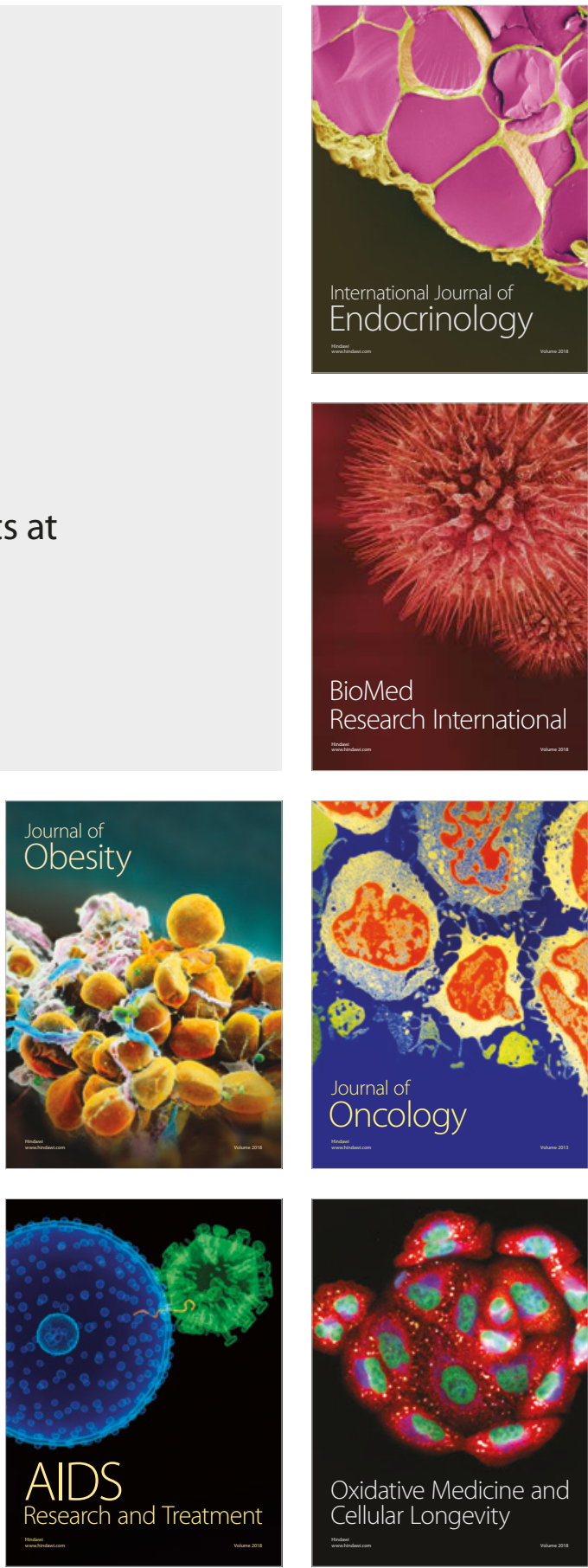